\title{
LA PISCICUltuRE EN DOMBES
}

\author{
par RÉgine LEVRAT
}

\author{
(suite) \\ II

\section{L'EXPLOITATION DES ETANGS}

L'exploitation des étangs de la Doimbes est restée ịnchangée pendant des siècles; actuellement encore elle est proche de ce qu'elle était à l'órigine. Elle demeure-basée sur l'assolement traditionnel, deux années d'évolage, une année d'assec. La seule modification importante a, été réalisée lors de l'introduction des Carpes de race sélectionnée, c'est le remplacement de la pêche à deux ans par celle à un an.

Pourtant, depuis quelques années, certains pisciculteurs dombistes s'efforcent d'améliorer le mode d'exploitation traditionnel par la pratique d'amendements qui leur permettent de prolonger l'évolage durant plusieurs années consécutives. Ils sont encore peu nombreux et leurs expériences ne portent que sur un faible pourcentage d'étangs.

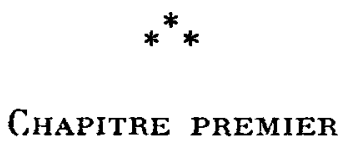

\section{L'EXPLOITATION TRADITIONNELLE DES ÉTANGS}

L'exploitation traditionnelle des étangs de la Dombes, représente un mode de mise en valeur original, que l'on ne trouve nulle part ailleurs, et dont on peut admirer aujourd'hui la remarquable adaptation aux données imposées par la nature et par l'histoire de la fondation dé ces nappes d'eau. Plus de $80 \%$ des étangs de la Dombes sont encore exploités de cette façon et, quel que soit son caractère routinier, ce mode de mise en valeur assure encore aux étangs de cette région malgré des conditions

(1) Voir Bulletin Français de Pisciculture, nos 215 et. 216 . 
naturelles particulièrement mauvaises, un rendement moyen supérieur à celui de l'ensemble des régions piscicoles françaises.

\section{La périodicité des grands travaux}

La vie de l'étang se déroule selon un double cycle, celui de l'assolement et celui de l'évolage.

L'assolement traditionnel, deux années d'évolage, une année d'assec, est rythmé par l'année d'assec qui permet à l'étang de se reposer et au pisciculteur d'effectuer les divers travaux que nécessite celui-ci. Jusqu'à l'introduction des Carpes de race sélectionnée, l'étang était pêché après les deux années d'évolage; actuellement il l'est toujours après une année seulement, permettant ainsi une seconde production.

Les différents travaux d'entretien de l'étang : curage des biefs, de la pêcherie, vérification du bon état de la chaussée, doivent être faits théoriquement chaque fois que l'étang est en assec ; pratiquement il est fréquent que les fermiers négligent de le faire pendant plusieurs assecs consécutifs. Avec les moyens actuéls, les travaux de curage peuvent attendre sans inconvénient 10,12 ou même 15 ans.

Le cycle de l'évolage est annuel, il est rythmé par la mise en eau et l'empoissonnage et surtout par la.vidange et la pêche de l'étang. Un certain nombre d'impératifs dictent le choix de la date de la pêche :

- Les coutumes réglementant les droits respectifs des différents propriétaires d'un même étang, bien que celles-ci tendent à disparaître avec la simplification du régime de la propriété ;

- Les nécessités culturales, l'année qui précède l'assec; l'avoine est semée au printemps, en mars ou avril, le blé doit l'être à l'automne. Les usages réglant les droits des propriétaires de l'assec et de ceux de l'évolage sont basés sur cette nécessité;

- Les exigences de l'élevage piscicole : il est préférable de pêcher le poisson durant les mois froids puisque celui-ci ne se développe que durant la belle saison. La pêche et le transport du poisson exigent des manutentions hors de l'eau que celui-ci ne supporte pas lorsque la température extérieure est élevée; dans ce cas on est obligé de pêcher de nuit avec des risques de pertes plus élevées;

- La demande du marché et les disponibilités des pêcheurs qui, peu nombreux, doivent pêcher un très grand nombre d'étangs, durant une période brève, avec un matériel limité et de faibles possibilités de stockage.

Tant que les usages anciens étaient de rigueur on pêchait du 1 er novembre au 31 mars, cette dernière date pouvant être avancée selon la coutume en vigueur dans la région (1). L'année de l'assec ne durait en

(1) Voịci ce que dit Bossr (op. c.) de la législation concernant la pèche : a On pêche depuis le $1^{\text {er }}$ novembre jusqu'au 31 mars; cependant on soutient que l'étang doit être à sec au 26 mars. Collet dit qu'il doit l'être au 15 ; quoi qu'il en soit, il conviendrait qu'il le-fut plus tôt encore ". 
fait que huit mois, de mars à novembre, ce qui, semble-t-il, ne lésait pas le propriétaire de l'assec car l'avoine seule était cultivée. Actuellement, la majorité des pêches ont lieu durant les mêmes mois que par le passé ; cependant, du fait des nécessités du marché et de l'amélioration des conditions de transport, cette période tend à s'étaler. Voici approximativement la répartition des pêches de la Coopérative au cours de l'année, telle que nous l'a indiquée oralement son directeur M. BEL :

\begin{tabular}{|c|c|c|c|}
\hline Octobre. & $18 \%$ & $\mathrm{a} \dot{\mathrm{p}} \hat{\mathbf{e}}$ & le tc \\
\hline Novembre.$\ldots \ldots \ldots$ & $37 \%$ & - & - \\
\hline Décembre $\ldots \ldots \ldots \ldots$ & $20 \%$ & - & - \\
\hline Janvier à mai & $20 \%$ & - & - \\
\hline Été & $5 \%$ & - & - \\
\hline
\end{tabular}

La mise en eau se fait généralement dès l'automne ou le début de l'hiver après la pêche ou. la levée des récoltes, afin de profiter des pluies de cette saison. Si, toutefois, l'étang peut être rempli plus tardivement après les premières gelées, grâce à des sources ou à l'eau d'un étang supérieur, cela ne peut avoir que des avantages, surtout dans le cas d'un étang de $1^{\text {er }}$ ou $2^{\mathrm{e}}$ été. L'étang doit être plein au début du printemps, en février s'il contient des Brochets.

L'empoissonnement se fait en automne ou durant l'hiver. Certains pisciculteurs estiment qu'il faut le faire dès que l'étang est en eau, afin de laisser au poisson le temps de "reconnaître les eaux " avant l'époque du frai. De plus en plus les pisciculteurs tendent à penser que le moment choisi importe peu, entre l'automne et le début du printemps, car l'hiver est une saison morte pour la croissance des poissons' et l'adaptation de ceux-ci est l'affaire de quelques jours. Ils empoissonnent lorsqu'ils disposent des meilleurs alevins, généralement tòt dans la saison s'ils achètent leur empoissonnage car ils ont alors plus de choix, souvent à des dates différentes pour chacune des espèces.

\section{La pratique de l'assec avec culture}

La pratique de l'assec est à la base de l'exploitation traditionnelle. des étangs de la Dombes; évolage et assec vivent en étroite symbiose; le séjour de l'eau amende un sol qui, sinon, serait inculte ; l'assec empêché l'usure du fond des étangs. L'assec avec culture représente pour l'étang. un amendement excellent qui contribue largement à expliquer les bons rendements des étangs de la Dombes. Ce mode d'exploitation, nous. l'avons remarqué, est original ; aucune autre région piscicole de France ou d'Europe, à notre connaissance, ne pratique l'assec ávec culture; par contre dans la plupart de ces régions, les étangs sont laissés èn assec lorsque cela est possible, une année de loin en.loin.

L'assec, même sans culture, à des propriétés bénéfiques pour:le fond de l'étang. Il " aère " le sol ; celui-ci, à l'air libre, se dessèche, se fissure, permettant ainsi à l'oxygène de le pénétrer profondément. Cét oxygène permet la minéralisation des matières organiques de la vase, 
travail qui est freiné ou bloqué si l'étang est toujours en eau. Cette minéralisation, sans laquelle la vase devient putride, est nécéssaire à l'équilibre biologique de l'étang; elle favorise. les échanges entre le fond et l'eau et élève le $p \mathrm{H}$ de .ceux-ci. Ce travail est activé par le labour qui assure une meilleure aération du sol. L'assec, d'autre part, élimine les parasites et lẹs agents: responsables des maladies, particulièrement lorsqu'il est renforcé par le gel, et permet le développement des vers, dont se nourriront les Carpes. L'expérience prouve que les étangs où il n'est pratiqué que tous les huit ou dix ans, ont une production qui s'accroît après chaque assec et diminue ensuite régulièrement.

La culture telle qu'elle est faite en Dombes améliore les vertus de l'assec, par l'apport d'engrais ét l'ameublissement du sol. La culture la plus habituelle est celle de l'avoine, de la variété dite " avoine grise des Dombes ", dont les rendements sont médiocres mais dont l'usage témoigne qu'elle améliore les pêches suivantes (1). Cette céréale se vend actuellement très mal; aussi la remplace-t-on souvent par le blé; grâce aux engrais les rendements en sont excellents, mais il épuise le sol et nuit à la production piscicole. D'autres cultures sont favorables au poisson : les légumineuses et le lotier. Le lotier est considéré comme la meilleure culture possible sur les fonds d'étangs, d'autant que généralement il se sème tout seul, mais il ne se développe que certaines années et seulement sur certains étangs, $1 / 3$ des étangs de la région environ (2).

Laissons, pour conclure ce paragraphe sur la pratique de l'assec avec culture, la parole à A. WurTz principal spécialiste français de la pisciculture en étang : «Malgré la pauvreté du sol, gràce à cette pratique culturale, qui est indiscutablement la plus rationnelle pour cette région, les étangs des Dombes peuvent être rangés parmi les meilleurs d'Europe sous les climats tempérés." (3).

\section{L'empoissonnement}

Un bon empoissonnement est le principe de base d'une bonne pèche. Cet acte élémentaire est souvent le seul que fait le pisciculteur pour son étang avant de pêcher celui-ci. Il est particulièrement important en Dombes où, après chaque assec, le fond est absolument vierge de tout poisson.

Différents éléments peuvent varier d'un empoissonnement à l'autre ; le chargement à l'hectare, la proportion des poissons de chacune des espèces, la grosseur ét la qualité des alevins. Des facteurs nombreux

(1) Cette céréale avait son rôle à jouer dans l'économie de la région, car la Dombes faisait un élevage important de chevaux demi-sang pour l'armée, élevage qui est en plein déclin.

(2) Le lotier velu est une légumineuse pouvant servir comme fourrage, ou mieux pour ses graines que l'on récolte en juillet-août. On ignore l'usage de ces graines qui sont vendues à un prix élevé aux Pays-Bas, en Allemagne et même en Amérique du Sud. La Dombes en récolte environ $60.000 \mathrm{~kg}$ par an, et les vend de 3 à $8 \mathrm{~F}$ le $\mathrm{kg}$.

(3) A. WURTz : -article cité. 
guident le choix du pisciculteur : la durée de l'évolage, la valeur piscicole de l'étang, et enfin la demande du marché. Une loi peut servir de basé à tout empoissonnement. "Selon la taille que l'on désire, il suffit de faire varier la densité du peuplement en fonction de la productivité des étangs " (1). La demande du marché doit donc être considérée comme le facteur déterminant; pour les Carpes, par exemple, elle porte sur des sujets de 1.200 à $1.300 \mathrm{~g}$; en deçà de $1.000 \mathrm{~g}$ ou au-delà de $1.500 \mathrm{~g}$ la vente est plus difficile. M. BEL' nous a expliqué qu'il calculait l'empoissonnement des étangs de la Coopérative de façon mathématique, compte tenu de la productivité de chacun, en fonction des marchés qu'il prévoyait pour le moment de leur pêche.

Quelle que soit la multiplicité de ces facteurs, des barèmes peuvent être indiqués qui servent de base aux éleveurs.

Jusqu'à l'introduction des espèces nouvelles de Carpe et tant que la durée de l'évolage était fixée à deux années consécutives, les normes étaient celles-ci, pour un hectare cadastre :

- Carpes : 1 cent, c'est-à-dire 160 têtes ou 80 paires de panots de $180 \mathrm{~g}$ environ.

- Tanches : 5 à $10 \mathrm{~kg}$ de tanchons de $100 \mathrm{~g}$ environ.

- Brochets : 6 à 8 brochetons de 50 à $100 \mathrm{~g}$ que l'on introduit dans l'étang la seconde année seulement.

- Blancs : aucune quantité n'est indiquée (2).

La quantité, la variété et la grosseur des blancs que l'on met est très variable selon que l'on désire ou non obtenir de la friture et selon la quantité de Brochets introduits dans l'étang.

Depuis que les étangs sont pêchés à 1 an, ces chiffres ont perdu toute signification. Les normes actuelles sont très inférieures. Voici les indications que nous a donnéés J. Pingeón concernant les étangs de la coopérative. Pour 1 hectare cadastre :

- Carpes : 100 têtes de panots.

- Tanches : $10 \mathrm{~kg}$, ce qui représente également une centaine de têtes.

- Brochets : 10 têtes de $100 \mathrm{~g}$.

- Blancs $\because 10 \mathrm{~kg}$.

Les chiffres indiqués par d'autres pisciculteurs sont voisins. (3).

Ces chiffres n'ont qu'une valeur'indicative: Certains étangs peuvent être. " chargés " davantage en Tanches parce qu'ils ont un· fond qui leur convient, d'autres en Brochets. Voici à titre d'exemple' l'empoissonnage

(1) H. DE VilletTe : "Carpiculture ". Cette loi concerne la Carpe mais peuit être généralisée: pour tous les poissons élevés.dans les étangs de la Dombes.

(2) G. Gúnaux : op. c.

(3) P. Mas : travail cité.

Pour 1 ha : Carpes : 100 têtes, 200 pour les meilleurs étangs. Tanches : 10 à $25 \mathrm{~kg}$ de tanchons de 1 ań pesant de $50^{\circ} 100 \mathrm{~g}$. Brochets : 5 à 10 brochetons de 50 à $100 \mathrm{~g}$ pour 100 Carpes. Blancs : 5 à $10 \mathrm{~kg}$ à l'ha.

M. Thevenard nous a dit les mêmes chiffres que ceux de ce rapport-sauf en ce qui concèrne le 'Brochet pour lequel'il indique : 5 à 6 filations. de $150 \mathrm{~g}$ pour 100 têtes de Carpes, plus $10 \%$ de Brochets dé 200 à 250 g pour la póse: Nous soulignon's cette précision que-d'autres personnes nou's ont ausisi donnée. 
qu'a adopté après quelques expériences M. J. Prenat dans son étang de Gouille à Mionnay. Cet étang, a une superficie de 15 ha cadastre, 13 ha eau.

- Carpes : 1.300 têtes, de poids variables selon les années.

- Tanchons de 2 étés : $90 \mathrm{~kg}$.

- Filatons : plus de $30 \mathrm{~kg}$, qu'il met généralement en deux fois, une première à l'automne, une seconde au printemps.

- Blancs : $140 \mathrm{~kg}$.

De nombreux pisciculteurs, la majorité probablement, chargent davantage, pensant que pour beaucoup récolter il faut beaucoup semer. Les Brochets rétablissent généralement l'équilibre, mais au détriment de l'éleveur, car l'empoissonnement est très onéreux.

La provenance de l'empoissonnement est très important pour garantir sa qualité et son adaptation, notamment en ce qui concerne les Carpes plus fragiles que les autres espèces. Il est souhaitable que les carpillons mis dans un étang proviennent tous du même élevage; par contrè, les pisciculteurs préfèrent avoir des brochetons de diverses sources. Les pisciculteurs exploitant un grand nombre d'étangs préfèrent produirent eux-mêmes leur alevinage; ils sont ainsi assurés de sa qualité et celui-ci leur revient moins cher. Les autres l'achètent, soit directement à des éleveurs voisins, soit à la coopérative s'ils sont adhérents, soit à des marchands de poissons qui possèdent généralement un certain nombre d'étangs d'empoissonnement ou servent d'intermédiaires. Ils ont intérêt a acheter des panots assez gros car le coût de ceux-ci est relativement moins élevé que celui des petits et les pertes sont plus faibles.

\section{La surveillance et l'entretien de l'étang}

L'étang n'exige qu'un minimum de soins; ceux-ci ne doivent cependant pas être négligés car ils sont très importants pour le rapport de l'évolage (1).

Le pisciculteur doit surveiller le niveau de son étang, spécialement lors du frai car le poisson pose sur les herbes du bord, et vérifiér constamment l'état des grilles fermant l'arrivée d'eau, la vanne et les ébies. Il doit éloigner les braconniers, détruire les nuisibles et lutter contre la végétation qui tend naturellement à devenir trop envahissante, surtout sur les bords de l'étang que l'on ne peut labourer lors de l'assec. Le pisciculteur doit faucher ces herbes ou les brûler si cela est possible lorsque l'étang est à sec, car elles diminuent la surface utile de la nappe d'eau et nuisent ainsi à la production piscicole; pour cette raison du reste, la seconde année de l'évolage est en général un peu moins productive que la première.

Des travaux d'entretien doivent aussi être faits durant l'assec,

(1) C'est une des raisons pour lesquelles le fermage de l'assec seul est de plus en plus abandonné; le fermier, n'étant pas intéressé par le revenu de la pêche, négligeait la surveillance et l'entretien de l'étang dont il était généralement chargé. 
afin d'assurer le bon fonctionnement du remplissage et de la vidange de l'étang. Le pisciculteur profite de cette période pour curer les canaux et la pêcherie, travail particulièrement long et difficile, facilité actuellement par l'emploi généralisé des tracteurs. Il vérifie aussi le parfait état de la chaussée et du thou (1).

\section{La pêche}

La pêche est en Dombes ce qu'est la vendange en pays vigneron, jour de'travail intense et de fête, jour où l'étang dévoile enfin son fruit. Sa préparation et son exécution exigent du. pisciculteur une longue expérience et un coup d'œil exercé, car elleś entraînent des risques de pertes considérables.

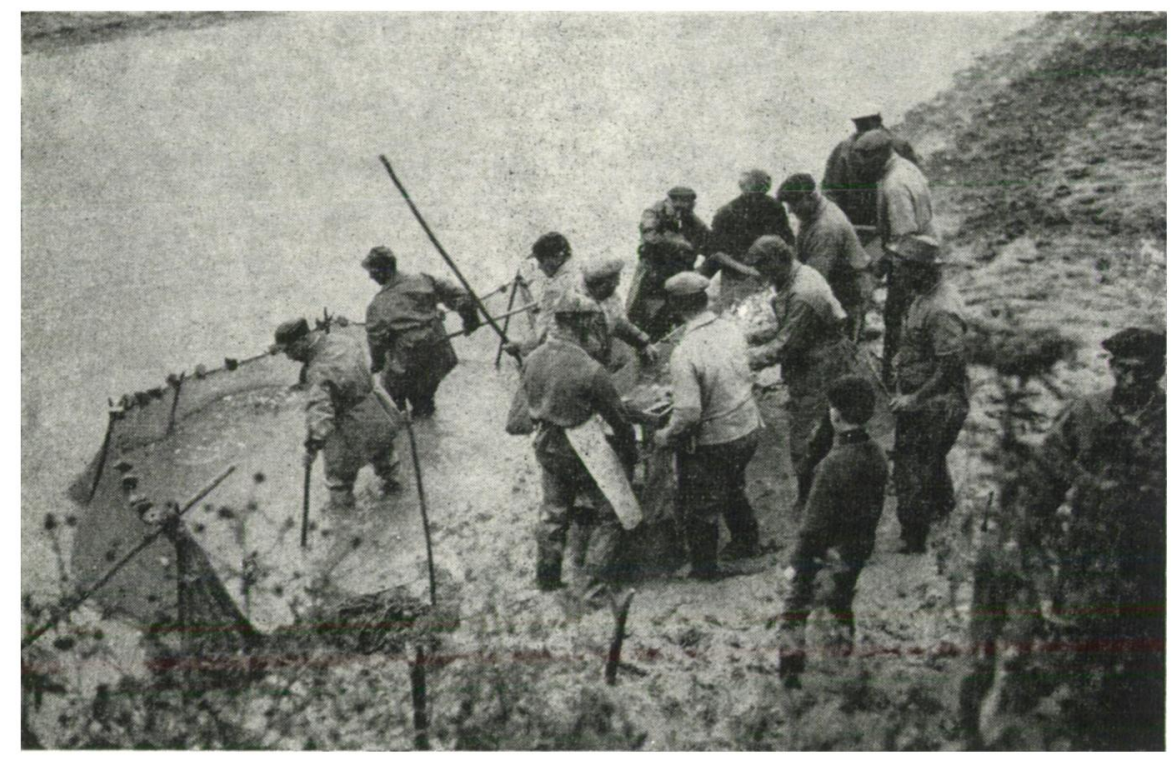

Bâtard (Senne ou Traine)

ramené en demi-cercle autour de la pécherie, après avoir été traîné le long du bief.

La date de la pèche est fixée longtemps d'avance entre le producteur et l'acheteur, car l'étang doit. être prêt, c'est-à-dire presque vide le jour dit. Quelques jours avant le moment prévu, quelques semaines pour les plus grands étangs, on ouvre la vanne, l'étang commence à se vider. Il ne doit se vider ni trop lentement afin d'être prèt à temps pour la pêche, ni trop rapidement, surtout si le temps est lourd, car le poisson rassemblé trop longtemps dans un cubage d'eau insuffisant risquerait de périr.

(1) Précisons que le labour pour la mise en culture se fait selon un procédé spécial, destiné à faciliter le drainage, c'est le labour en a planches ^ou " billons ». Les planches ont 90 a $100 \mathrm{~cm}$ de large, elles sont bombées par le milieu, un chenal d'écoulement est ménagé toutes les 6 raies environ. 
Tous les calculs peuvent être déjoués par de fortes pluies; il arrive même que l'ọn soit obligé de retarder la pêche, ce qui est très gênant à un:moment où les marchands de poissons ont généralement un programme très chargé. Durant les dernières heures il faut surveiller l'étang de très près, ralentir ou accélérer la vidange, éloigner les prédateurs et maraudeurs; un veilleur assure toujours la garde sur les rives de l'étang à ce moment-là.

La pêche exige un personnel nombreux et compétent car elle doit se dérouler le plus rapidement possible. Le marchand de poisson ou la coopérative amènent toujours quelques spécialistes, les autres pêcheurs sont recrutés sur place et payés en nature. Elle demande d'autre part un matériel adapté. Celui-ci comprend :

Deux sortes de filets :

- Un grand, le "bâtard ", encore appelé " senne " ou " traine », de 20 à 25 mètres de long, avec une nappe d'une largeur de 1,8 à $2 \mathrm{~m}$, àux mailles larges, de 1,5 à $2 \mathrm{~cm}$, destiné à ramener dans la pêcherie les gros poissons ;

- Quelques filets plus petits, aux mailles plus fines, les " seillettes » ou seyettes qui servent à pêcher les alevins.

- Des épuisettes de deux types :

- Les unes très fortes, les " arvouds ", qui peuvent contenir une dizaine de $\mathrm{kg}$ de poisson. de pêche.

- Les autres plus légères, les "troubles ", servant surtout en fin

- Des filochons pour le transport du poisson, capables de supporter une charge de 20 ou $30 \mathrm{~kg}$.

- Une table de triage -ou " gruyère ", long couloir en bois de $3 \mathrm{~m}$, sur $0,50 \mathrm{~m}$, ouvert à l'une des extrémités pour permettre l'écoulement du poisson.

- Une balance de type b̦alance romaine.

La pêche a toujours lieu;le matin, car de nuit le poisson rassemblé dans très' p'eu d'eau résistë mieux au risque d'asphyxie qu'il èncourt durant les dernières heures d'attente. Elle commence généralement à l'aube ou même avant le lever dú soleil lorsque l'étang est grand ou le programme de la journée chargé. Elle est réalisée en quelques heures; il est possible, si tout marche normalement, de pêcher avec une douzaine d'hommes $5.000 \mathrm{~kg}$ de poissons en deux heures (1). Les étangs de la Dombes, sauf les deux ou trois plus grands, peuvent être pêchés dans la demi-journée. Durant les périodes plus chargées, les marchands arrivent à effectuer deux, parfois trois pêches dans la matinée.

La pêche se déroule en quelques opérations : le coup de filet, la pêche, le tri, le pesage du poisson. Quelques hommes trainent le "bâtard "le long du bief central, de l'amont vers l'aval ramenant ainsi le poisson vers la pêcherie. Le filet est rapproché de la rive en un demi cercle, que l'on resserre au fur et à mesure de la pêche; il est maintenu à l'aide de

(1) P. MAS : travail cité. 
fourches de bois, plantées dans le sol. Quelques hommes, équipès de bottes cuissardes, pêchent alors le poisson prisonnier avec les épuisettes et le déversent dans la table de triage installée tout près. Les hommes alignés le long de la gruyère trient le poisson et le répartissent dans les filochons que les porteurs tiennent appuyés en face d'eux. Ceux-ci le transportent jusqu'à la balance où se tiennent, carnet en main, le vendeur et l'acheteur, puis une fois pesé, jusqu'à l'un des camions du marchand. Là, un homme hisse les filochons, répartit le poisson dans les différents bacs et surveille celui-ci.

Deux ou trois coups de filet avec le bâtard sont parfois nécessaires, puis quelques coups encore avec les seyettes.

La pêche achevée, la fête commence, fête pour les pêcheurs, fête aussi pour les oiseaux qui, une fois le champ libre, s'empressent de profiter des reliefs.

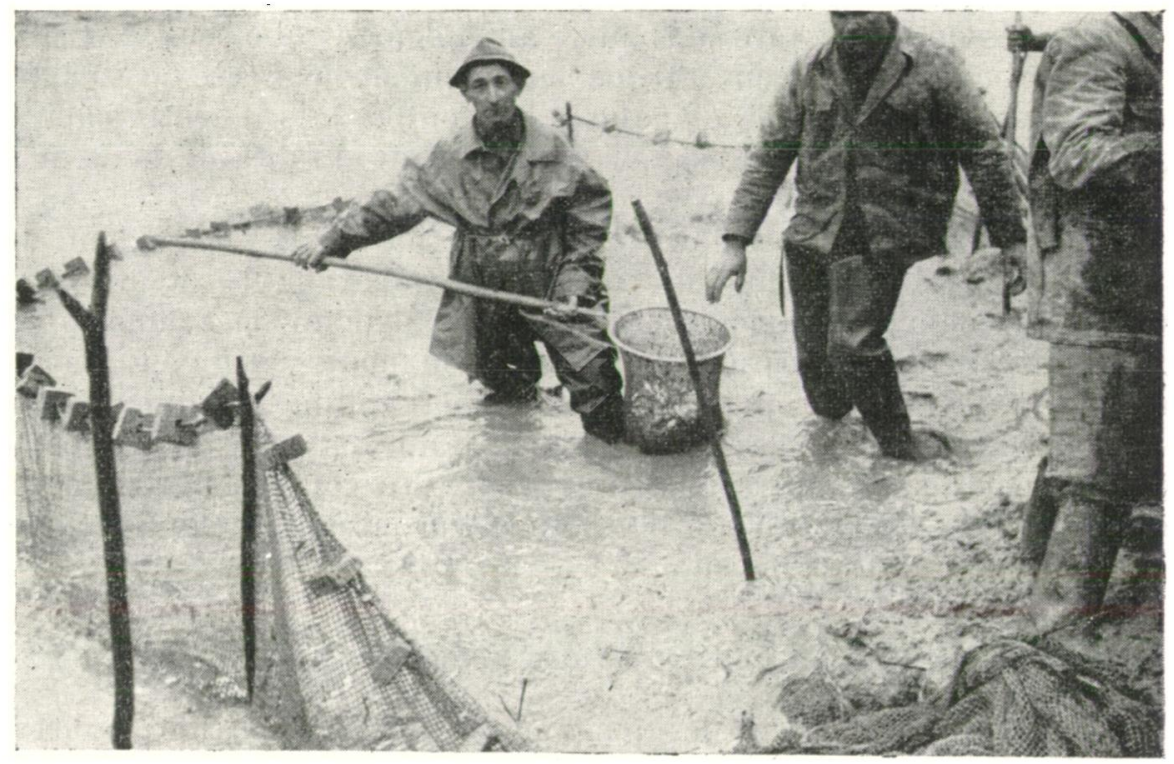

La pêche au moyen de l'arvoud

\section{Chapitre II}

\section{LES REGHERGHES RECENTES ET LES POSSIBILITÉS NOUVELLES D'EXPLOITATION}

Les recherches concernant la pisciculture sont récentes en France ce qui s'explique én Dombës par l'excellence de l'exp̈loitation traditionnelle et sur le plan nationál par la faible importance'du-secteur piscicole par rapport aux autres secteurs agricoles. Elles ont porté d'abord sur-la sélection des espèces élevées, la Carpe notamment'; elles ne pórtent que depuis peu sur l'amélioration du milieu naturel; l'étang. Ces travaux 
sont l'œuvre, en Dombes de quelques pisciculteurs avisés qui, depuis le début du siècle et surtout depuis la dernière guerre, s'efforcent d'améliorer l'exploitation traditionnelle de leurs étangs, et, sur le plan national, du Service d'Hydrobiologie appliquée des Eaux et Forêts. En Dombes, des recherches théoriques ont été faites sur la valeur piscicole des étangs ; des amendements nouveaux ont été expérimentés qui permettent d'envisager un mode d'exploitation différent.

\section{La capacité biogénique des étangs}

On entend par capacité biogénique d'un ètang sa valeur théorique au point de vue piscicole ou, de façon plus pratique, la quantité de poisson qu'il peut nourrir à l'hectare.

Il est aisé d'estimer approximativement la capacité biogénique d'un étang; en Dombes certains étangs produisent bon an mal an 200 ou $250 \mathrm{~kg}$ de poisson à l'hectare sans autre amendement que celui de l'assec, d'autres par contre atteignent à peine le rendement médiocre de $80 \mathrm{~kg} / \mathrm{ha}$. Il est beaucoup plus difficile d'analyser les composants de cette capacité biogénique, autrement dit de savoir pourquoi un étang a de bons ou de mauvais rendements.

Nonobstant quelques éléments dont le rôle vital est évident, tels que l'abondance de l'eau dont il dispose, l'oxygénation et la température de celle-ci, la capacité biogénique d'un étang dépend de la quantité et de la qualité de la nourriture que le poisson peut y trouver, puisque celui-ci se développe proportionnellement au volume d'aliments qu'il assimile. Deux problèmes se posent alors ; d'une part, de quoi se nourrissent les poissons; d'autre part, quels sont les composants chimiques et organiques du sol et de l'eau d'un étang qui permettent aux éléments nutritifs de celui-ci de se développer.

\section{La nourriture des poissons}

De quoi $\& \geq$ nourrissent les poissons? A cette question, pourtant élémentaire, il est difficile de répondre (1). Nous étudierons essentiellement l'alimentation de la Carpe sur laquelle ont porté la plupart des études et qui peut être considérée comme la base de celle de l'ensemble des cyprinidés; nous introduirons au fur et à mesure les nuances propres à chacune des espèces.

La Carpe est un omnivore à prédominance carnivore. Voici ce que dit P. Vivier de son alimentation : "On a dit que la Carpe était " le porc" de l'étang : elle est, en effet, peu difficile sur le choix de ses aliments. On a trouvé parfois dans son estomac une nourriture uniquement végétale, composée de petits fragments de feuilles, de tiges, de racines, d'algues, de graines de graminées et même de lentilles d'eau. Elle est surtout carnassière et s'attaque essentiellement aux invertébrés, bien qu'elle soit

(1) Nous ne parlerons ici que de l'alimentation des cyprinidés, à l'exclusion des piscivores pour lesquels le problème est différent et ne se pose qu'à propos de l'empoissonnement. 
susceptible de manger parfois quelques poissons, ses propres alevins - et même des grenouilles. Mais c'est le plancton et le benthos des rives ou du fond qui constituent sa nourriture de base "(1). Le menu des Carpes peut donc varier selon les ressources de l'étang, la. saison et le caprice de l'heure. Au printemps les Carpes trouvent dans le sol, surtout aprèsl'assec, un grand nombre d'animaux hivernants puis, les plantes aquatiques commençant à pousser, elles se nourrissent d'une multitude d'insectes que celles-ci abritent; un peu plus tard lorsque le plancton se dévelcppe à son tour elles le choisissent de préférence. Enfin ces différentes possibilités épuisées, elles s'alimentent aux dépens du benthos. A part le Gardon, à prédominance herbivore, tous les cyprinidés ont pour plats de résistance le benthos et le plancton.

- Le benthos est composé d'algues, de diatomées, de protozoaires, de bactéries et d'invertébrés microscopiques, de vers, de larves d'insectes, de mollusques... Il est moins abondant dans les étangs de Dombes qui subissent régulièrement l'assec que dans ceux qui sont continuellement en eau ; il est cependant assez riche. La Tanche èst essentiellement benthophage, la Carpé a une préférence pour le plancton.

- Le plancton comprend des végétaux : c'est le phytoplancton, et des animaux : c'est le zooplancton. Le phytoplancton est composé surtout de diatomées, de péridiniens et de flagellés.. Le zooplancton est formé de protozoaires, de rotifères, d'entomostracés. Les. Carpes consomment essentiellement le zooplancton qui se nourrit lui-même de phytoplancton. Le développement du plancton se déroule selon un cycle : le phytoplạnctón commence à pousser au début du printemps grâce à la chaleur et à la lumière solaire; il se multiplie rapidement, puis le zooplancton se forme au détriment de celui-ci. Le maximum du zooplancton a lieu en mai-juin. Ensuite le phytoplancton affaibli par le zooplancton reprend sa progression et permet un renouveau de zooplancton vers septembre, octobre. L'importance du plancton est telle pour la nourriture des Carpes que l'on a pu établir une classification des étangs au point de vue piscicole en fonction de leur indice planctonique (2). Cependant, quelques-uns mis à part, les étangs des Dombes sont pauvres en plancton; ils ont pourtant une production piscicole assez bonne, bien supérieure à celle que l'on pourrait prévoir d'après cette classification. Il semble que, faute de plancton, les Carpes de la région consomment surtout du benthos.

\section{Valeur biogénique des eaux et des sols des étangs}

La quantité et la qualité de la nourriture qu'un poisson peut trouver dans un étang dépendent de la richesse de son sol et plus encore de son eau. Aucun rapport n'a pu être établi entre ces deux données; cependant

(1) Le " plancton " groupe' "l'ensemble des êtres microscopiques qui flottent passivement ou non dans l'eau $»$. Le « benthos " comprend "l'ensemble des êtres, végétaux et animaux, vivant sur le fond du lac, ou à l'abri des végétaux aquatiques ". P. Vivier : La vie dans les eaux douces. 1921.

(2) L. LEgER : Travaux du laboratoire de pisciculture de l'université de Grenoble, 
on connaît de façon empirique la valeur piscicole de quelques éléments chimiques et organiques constitutifs des eaux et des sols des étangs :

- L'importance du $p \mathrm{H}$. Un bon étang est basique. On estime en règle générale qu'il doit avoir un $p H$ compris entre 7 et 8 .

- La nécessité d'une teneur élevée en chaux et en phosphore. Le phosphate de chaux forme l'armature du squelette des poissons. La tonne de Carpes que produit annuellement un étang cultivé de 10 ha enlève $10 \mathrm{~kg}$ de chaux par an à celui-ci (1). algues.

- Le rôle de la silice et du fer dans la multiplication de certaines

- La valeur des matières organiques qui permettent le développement de la faune de l'étang.

"La chaux, l'acide phosphorique et l'azote nitrique décroissent dans les étangs, en même temps que la saison s'avance, car ils sont absorbés au fur et à mesure de leur production par les êtres vivants " (2). Ceci est bien la meilleure preuve de leur utilité. La nécessité de chacun de ces éléments est reconnue, l'insuffisance de l'un d'entre eux ne peut être compensée par l'abondance des autres. Une loi dite du minimum a été ainsi formulée : "L'abondance des organismes est proportionnelle à la quantité de l'élément fertilisant absorbable et assimilable qui se trouve au minimum, relativement au besoin de ces organismes " (3).

Les étangs de la Dombes sont riches en silice et en fer; par contre ils manquent de chaux et de phosphore et ont un $p \mathrm{H}$ très faible. La teneur de leurs eaux en matières organiques est très variable.

D'après ces diverses données concernant l'analyse de la nourriture du poisson et celle des composants chimiques et organiques de l'eau et du sol des étangs, les étangs des Dombes devraient avoir une capacité biogénique de l'ordre de $60 \mathrm{~kg} / \mathrm{ha}$; or en fait elle est de plus du double.

\section{Les recherches actuelles}

Des recherches scientifiques ont été faites récemment par le Service des Eaux et Forêts sous l'égide de M. Vivier, Directeur de la Station Centrale d'Hydrobiologie appliquée à Paris; elles sont l'cuvre de A. Wurtz, alors Directeur de la Station d'Hydrobiologie du Paraclet dans la Somme, assisté de M. PINGEON pisciculteur dombiste particulièrement averti, président du Syndicat d'Étangs de la région (4). Ces divers travaux n'ont pas donné tous les résultats que l'on en attendait; ils n'ont pas permis de résoudre le problème que pose la capacité biogénique des étangs de la Dombes mais seulement de le mettre en évidence avec plus de relief et de complexité.

(1) J. Duché : cité par P. Vivier : La vie dans les eaux douces.

(2) P. Vivier : idem.

(3) Liebrg : cité par P. Vivier : idem.

(4) Nous avons pu avoir des renseignements sur les expériences de A. Wurtz par Mme Wurtz qui l'a assisté dans ses travaux, par M. Vivier et par M. Pingeon, ainsi que par le compte rendu qu'en donne M. Mas dans son étude. 
A. Wurtz a suivi pendant 5 ans une douzaine d'étangs des Dombes, de mars à novembre, trois jours par mois. Ses analyses ont porté sur :

- Les. sols ·des étangs ;

- La valeur piscicole des eaux (1);

- Le plancton : constituants et volume;

- Diverses observations sur le poisson : maladies, croissance, alimentation, contenus stomacaúx...

A. Wurtz avait poursuivi des expériences analogues dans de nombreuses régions piscicoles de. France, expériences qui l'avaient àmené à des conclusions assez précises, notamment sur le rapport entre l'indice planctonique et la production de poisson. Il pensait tout naturellement que les analyses faites en Dombes confirmëraient ses conclusions' antérieures. Le résultat de ces analyses a été déroutant (2); non seulement il n'a pas permis dé résọudre le problème de la capacité biogénique des étangs des Dombes mais il a souvent infirmé celui de tous les travaux précédents concernant. d'autres régions. Des étangs -chimiquement et biologiquement très riches ne produisent pas forcément plus, mais parfois moins même que d'autres plus pauvres. Il n'est pas possible d'établir un rápport constant entre l'indice planctonique des étangs et leur production. Parällèlement un, autre fait, demeure inexpliqué: les Carpés étudiées sont maigres en début de saison, période où le plancton abonde, elles sont grasses en septembre alors que celui-ci s'appauvrit.

Voici les conclusions de M. PIngeon sur les travaux qu'il a effectués avec A. Wurtz :

"Malgré une accumulation de documents, aucune directive n'a encore pu être dégagée de ces étụdes. Le problème de la productivité des étangs de Dombes resté encore inexṕliqué. Les |conclusions résultant de l'étude semblable des étangs des autres régions piscicoles de France : Brenne, Sologne, Est, ne se retrouvent pas en Dombes. Les résultats actuels de toutes ces analyses sont assez décevants : les étangs dont les eaux sont chimiquement les meilleures (au point de vue piscicole), ne sont pas les plus, productifs. Au contraire, les étangs les meilleurs ont des données chimiques ne dépassant pas la moyenne de celles des autres étangs. La productivité des très bons étangs de Dombes tient donc à là présence d'un micro-élément qu'il s'agit de découvrir " (3).

Ouvrons une parenthèse avant d'achever ce. paragraphe sur les recherches actuelles en Dombes pour signaler une autre expérience faite par M. Pingeon portant non plus sur la capacité biogénique des étangs des Dombes mais tendant. à expliquer les variations de production de cette région, d'une année à l'autre, variations très importantes, en fonction des variations climatiques. M. Pingeon a tracé des courbes extrêmement précises des températures moyennes maxima et minima de

(1) Température de l'eau, couleur, turbidité, oxygène dissous, B. O. D., conductivité, résidu sec, matières organiques, $\mathrm{CO}_{2}$ ţotal, degré hydrotimétrique, alcalinité, calcaire, $\mathrm{Mg}, \mathrm{P}, \mathrm{Na}$, chlorures, nitrates, $\mathrm{P}_{2} \mathrm{O}_{5}$.

(2) Voir infra, annexe 2.

(3) J. Pingeon : article cité. 
chaque mois, durant un grand nombre d'années et a dessiné également des courbes des rendements en poisson, en Carpes notamment, des étangs de la coopérative durant ces mêmes années (1). Aucun rapport n'a pu ètre établi entre celles-ci.

\section{Les amendements}

La pratique de l'asșec a été pendant des siècles le seul amendement procuré aux étangs de la Dombes; elle le reste dans la plupart des cas ; cependant quelques exploitants s'efforcent d'améliorer cette pratique par l'apport d'engrais et par l'emploi de nouvelles techniques qui permettent de lutter contre l'excès de végétation.

Il est possible d'accroître la capacité biogénique d'un étang par l'apport d'engrais qui compensent l'absence de certains éléments chimiques ou organiques des étangs. Au $x_{1 x}{ }^{e}$ siècle, quelques novateurs ont réalisé avec succès le chaulage de leurs étangs, ils n'ont cependant pas fait école. Depuis la dernière guerre un certain nombre de propriétairesexploitants font des expériences d'engrais divers, ils sont arrivés à améliorer de façon notable les rendements de leurs étangs. Ces engrais sont générälement épandus sur l'cau et visent à enrichir d'abord celle-ci puis par contre coup le sol. Ils doivent être mis de préférence en plusieurs fois. Les principaux amendements utilisés sont :

- La chaux : Elle est la substance la plus nécessaire aux étangs de la Dombes. Elle désacidifie le milieu, élève le $p H$, accélère l'activité des bactéries et assure ainsi une meilleure minéralisation et nitrification de l'eau. Le chaulage se traduit généralement par l'accroissement du planc ton. La production de poissons augmente de façon sensible après un apport suffisant; on peut citer l'exemple d'étangs dont le rendement est passé de 150 à $300 \mathrm{~kg} / \mathrm{ha}$; cependant la chaux étant peu soluble, cet apport doit être massif si on veut en avoir le bénéfice dès la pêche suivante. Le chaulage peut se faire sous différentes formes, chaux vive ou éteinte ou encore calcaire broyé de plus en plus employé actuellement. La quantité apportée était il y a quelques années de 200 à $600 \mathrm{~kg} / \mathrm{ha}$ de chaux éteinte, mais celle-ci s'avère insuffisante. $M$. BEL nous a indiqué comme norme annuelle le chiffre de 500 à $1.000 \mathrm{~kg}$ de calcaire broyé par hectare (2). L'épandage doit se faire de préférence sur l'eau, au printemps.

- Le phosphore : Il est après la chaux la substance la plus nécessaire aux étangs. Son apport accroît toujours leur rendement en poisson;

(1) Les températures choisies sont celles de la Station météorologique la plus proche, celle de Bron. Les étangs de la coopérative représentent environ le $1 / 4$ de la superficie en eau des Dombes.

(2) Nous signalons à ce propos une formule établie par M. P. Chrmirs, précisée par M. P. Thieny et quẹ cite M. P. Vivier dans La vie dans les eaux douces; $\mathrm{k}$ désigne la quantité de chaux vive à apporter, h la profondeur de l'étang à la bonde, DH le degré hydrotimétrique, le chiffre de 15 étant considéré comme l'indicateur d'une eau piscicolement bonne : $k=20$ h $(15 \mathrm{DH})$. 
il est fait en Dombes sous forme d'hyperphosphates et surtout de scories Thomas. J. Pingeon indique comme quantité de base l'emploi de $30 \mathrm{~kg} / \mathrm{ha}$ de $\mathrm{P}_{2} \mathrm{O}_{5}$ (1).

- L'azote et le potasse : Leur apport semble souvent efficace bien que de façon moins manifeste que celui de, la chaux ou du phosphore. Il n'est cependant pas toujours à propos, car azote et pótasse activent la croissance aussi bien des plantes parasitaires que de celles qui sont utiles. Ces, deux engrais doivent être utilisés à bon escient car en cas de sécheresse le développement excessif des plantes émergées peut nuire gravement aux pêches consécutives.

- Les fumures : L'apport de fumures à l'étang est extrêmement bénéfique bien que celles-ci n'apportent qu'une amélioration passagère. Il est rare en Dombes qu'un exploitant apporte du fumier dans son étang car il le réserve aux cultures; cependant beaucoup d'étangs reçoivent de l'eau qui en est riche ou sont pâturés par du bétail.

Ces différents engrais améliorent la production piscicole en favorisant le développement des micro-organismes dont se nourrit le poisson. Peu d'étangs en bénéficient actuellement, mais les expériences réalisées prouvent de manière concluante qu'il est possible d'accroître ainsi de façon très importante le rendement des étangs. (2).

Une autre tentative a été faite en Dombes : nourrir artificiellement, en tout ou en partie, les Carpes, par l'apport d'aliments tels que le maïs (3). Cette méthode s'est généralisée dans divers pays, notamment en Yougoslavie. Les Carpes nourries ainsi acquièrent en deux ans un poids au moins égal à celui obtenu naturellement en trois ans ou plus. Les étangs exploités de cette façon en Yougoslavie, qui, par ailleurs sont naturellement excellents, ont des rendements environ 10 fois supérieurs à ceux de la Dombes. Cette opération ne peut cependant être-rentable en France dans les conditions de vente actuelles ; son succès dans d'autres pays prouve cependant qu'elle peut devenir intéressante. Ce procédé représente peut-être l'avenị de la pisciculture en Dombes.

Un autre amendement est plus répandu en Dombes que celui qui consiste à apporter des engrais à l'étang'; c'est là lutte contre les plantes émergées ou flottantes qui permet d'accroître la surface utile de l'étang et éventuellement de prolonger l'assec sans que pour autant la nappe d'eau soit envahie par les végétaux. Elle peut se faire par des procédés mécaniques tels que le faucardage ou par l'emploi de produits chimiques.

Le faucardagè consiste à couper les plantes sous l'eau avec un bateau-faucheur ou faucardeur. Celui-ci est une simple barque à moteur équipée à l'avant de deúx faux, l'une horizontale qui fauche les plantes à 15 ou $20 \mathrm{~cm}$ de la surface, l'autre verticale qui permet à l'engin de se frayer un passage à travers les herbes. La meilleure saison pour faucarder

(1) J. Pingeon : article cité.

(2) Voir infra, l'évolution deș rendements de deux étangs auxquels on a procuré des engrais.

(3) Après des expériences de produits divers, il semble que le maïs soit le plus rentable. Son quotient nutritif est de 4 ou 5 . 
est celle où la végétation se développe le plus, au mois de juin. Le faucardage a été expérimenté en Dombes depuis une quarantaine d'années ; sa pratique se généralise depuis 10 ou 15 ans, grâce à la coopérative qui met des bateaux à la disposition de ses membres. Ce procédé est particulièrement efficace contre les roseaux qu'il élimine de façon durable; il agit moins vigoureusement sur les plantes flottantes qui repoussent après chaque coupe. On commence à lutter contre celles-ci, particulièrement contre la mâcre ou chataîgne d'eau par l'emploi d'un. désherbant chimique épandu lors de l'assec, un sel de sodium, le 2.4 D qui favorise le développement du plancton et ne semble nuire au poisson (1).

\section{Les possibilités nouvelles d'exploitation}

L'apport d'engrais à l'étang en eau enrichit celui-ci, d'autres part la pratique du faucardage évite l'envahissement de l'étang par la végétation. Ces nouveaux amendements permettent de remettre en cause l'assolement traditionnel car l'assec n'apparaît plus indispensable à l'évolage. Voici ce que dit à ce propos M. BEL : "L'assolement triennal était vital, il est devenu une hérésie ".

Actuellement les exploitants qui apportent des engrais à l'évolage prolongent la durée de celui-ci.jusqu'à 6 ou 8 années consécutives, parfois même plus. Il semble que l'on puisse en procurant à l'étang les amendements nécessaires, supprimer complètement la pratique de l'assec avec culture. Il faut toutefois dans ce cas laisser l'étang vidé le temps nécessaire pour les travaux d'entretien, quelques mois durant l'hiver si l'étang peut-être rempli au printemps, pendant l'été sinon. Avec les engins modernes qui permettent le labour de fonds très envasés et le curage de biefs plus profonds, il devient possible d'espacer les travaux et de les renouveler tous les 10 ou 12 ans seulement.

Des arguments d'un ordre différent mais non moins déterminants incitent les pisciculteurs à renoncer à l'assec avec culture : les difficultés de main-d'œuvre d'une part surtout pour les propriétaires non exploitants, la chasse d'autre part. Actuellement de nombreux propriétaires abandonnent la pratique de l'assec même s'ils n'apportent pas d'engrais en contre-partie à leurs étangs; ceci se fait évidemment au détriment de la production piscicole mais témoigne de la défaveur qui se dessine pour la mise en culture de l'étang. D'autres exploitants fidèles encore à l'assec, espacent celui-ci et mettent leurs étangs en culture tous les 4 ou 5 ans, environ. Quelques-uns toutefois désireux de faire pousser du blé sur leur fond d'étang, mettent celui-ci en culture deux années consécutives, ce qui, si l'on ne veut pas sacrifier la pêche précédente, est nécessaire :

- la première année est consacrée à une céréale de printemps;

(1) Le 2.4. D n'agit pas contre les roseaux; cependant IVurtz signale d'autres produits efficaces contre ceux-ci, le Nata, le Dowpon, le Telwar ou Monuron, enfin le Phragmitox, produits qui à notre connaissance ne sont pas utilisés en Dombes. A. Wurtz : article cité. 
- La seconde année au blé que l'on peut ainsi semer à l'automne. Chaque année cependant quelques pisciculteurs renoncent à mettre en culture leurs étangs.

L'assolement traditionnel se modifie au fur et à mesure que disparaissent les coutumes et usages anciens et que les étangs deviennent indépendants. Il est vraisemblable que d'ici quelques années la pratique de l'assec avec culture sera considérée comme périmée en Dombes. 